\title{
NEO-related scientific and outreach activities at KLENOT
}

\author{
Jana Tichá, Miloš Tichý and Michal Kočer \\ Klet' Observatory \& KLENOT Project, Zátkovo nábřeží 4, CZ-370 01 České Budějovice, \\ South Bohemia, Czech Republic \\ email: klet@klet.cz
}

\begin{abstract}
In the recent times, there has been a noticeable increase in interest about NEOs. In the light of results of recent NEO surveys the need for continuous follow-up astrometry to secure the orbits of the discovered bodies has risen.

The Klet Observatory Near Earth and other Unusual Objects Observation Team and Telescope, project KLENOT, started in 2002. It is dedicated to confirmation, follow-up, and recovery of NEOs. For this task the 1.06-m KLENOT telescope, equipped with a high-efficiency CCD is used. Since it was set in service in March 2002, a significant number of results have been obtained. For instance, 10,000 positions have been determined, more than 400 confirmations made, including 16 recoveries and 104 Virtual Impactors measured.

An important part of NEO search is the discovery of comets. Therefore an inseparable component of NEO follow-up includes the detection of possible cometary features of newly discovered bodies and the confirmation of Near Earth Comets (NEC).

In the next decade, surveys will be characterized by several aspects. Pan-STARRS opens the question whether "classical" confirmation and early follow-up of newly discovered NEO candidates will be necessary by others, and we discuss the need for possible changes in existing system of follow-up process. The most important challenge for follow-up will likely become the NEOs which are in urgent need of astrometric positions over longer arcs including Virtual Impactors, radar, and mission targets. The observing strategies and obtained results of the KLENOT Project will be presented as well as future plans will be discussed.

One of the most important duties of NEOs scientists and research institutions is to maintain contact with the public. NEO related issues have outstanding educational value and outreach potential. Nowadays the Internet has proved itself to be an excellent mean of bringing NEO knowledge to a wider audience.
\end{abstract}

Keywords. Astrometry; follow up; telescope; outreach

\section{Introduction}

Mainly due to increased interest from the scientific community and fortunately also from the general public, a tremendous work has been done in the study of NEOs in the last decade. Although, at least according to our present knowledge, we are not living under immediate danger of collision with potentially hazardous object, we have to carefully consider our response to such threats. Therefore the orbit determination with high precision is imperative. The task is, of course, more difficult and complex then it can be seen at the first sight. In its complexity the astrometry of NEOs is important but often overlooked activity. This paper presents some of the methods and procedures which contribute to reliable orbit determination of NEOs. The importance of follow-up astrometry including recovery in the second opposition is particularly stressed. 


\section{Klet' Observatory}

The Klet Observatory is located in the Czech Republic in central Europe.

The IAU/MPC observatory codes are (046) Klet Observatory, České Budějovice and (246) Klet Observatory-KLENOT. The geographical position of the observatory is longitude $\lambda=14^{\circ} 17^{\prime} 17^{\prime \prime} E$, latitude $\phi=+48^{\circ} 51^{\prime} 48^{\prime \prime} N$ and elevation $=1069 \mathrm{~m}$. There are about 180 clear nights per year with best weather conditions in February, March, August, September and October.

\section{KLENOT Project}

\subsection{KLENOT telescope}

The KLENOT telescope was built using an existing dome and infrastructure of the Klet Observatory. The original mounting was upgraded and the optoelectronical control system was added. A new control and computer room was built. All observing time belongs to the KLENOT team.

Optical system consists of 1.06-m f/3 main mirror (Sital glass, Carl Zeiss Jena) and four lenses primary focus corrector computed by Sincon, Turnov, Czech Republic and manufactured at the Optical Facility of Charles University, Prague, Czech Republic. It yields a $1.06-\mathrm{m} \mathrm{f} / 2.7$ optical system.

For the KLENOT telescope we use CCD camera Photometrics Series 300 with chip SITe 003B 1024 x 1024 pixels, pixel size 24 microns, liquid nitrogen cooling and Q.E. > 80 per cent in range $550-800 \mathrm{~nm}$ and $>60$ per cent in range $370-880 \mathrm{~nm}$.

Field of view is $33 \times 33$ arcminutes. Image scale is 1.9 arcseconds per pixel.

The limiting magnitude is $m_{V}=22$ for 180 -sec exposure time in standard observing conditions.

\subsection{KLENOT Goals}

- Confirmatory observations of newly-discovered, faint NEO candidates Some of new search facilities produce discoveries fainter than $m_{V}=20$ (for example 1.8-m Spacewatch II, 1.5-m Mt. Lemmon Survey) which need a larger telescope for confirmation and early follow-up. A 1-m class telescope is also very suitable for confirmation of very fast moving objects because larger aperture helps minimize trailing losses and our larger field of view enables us to search for NEO candidates having a larger ephemeris uncertainty.

- Follow-up astrometry of poorly observed NEOs

It is necessary to observe newly discovered NEOs in a longer arc during the discovery opposition often as they are getting fainter. Special attention is given to "Virtual Impactors" and PHAs, targets of future space missions or radar observations. On the other hand, it is necessary to find and use an optimal observing strategy to maximize orbit improvement of each asteroid.

- Recoveries of NEOs in the second opposition

For the determination of reliable orbits it is required to observe asteroids in more then one opposition. If the observed arc in a discovery apparition is long enough, the chance for a recovery in the next apparition is good. If the observed arc at single opposition is not sufficiently long, a larger uncertainty in its orbit determination requiring a search along the line of variations. For this purpose our larger field of view is a clear advantage.

- Follow-up astrometry of other unusual objects

We plan to make follow-up astrometry of other unusual objects, such as Centaurs and transneptunian objects, both at discovery and subsequent apparitions. To obtain positions of brighter transneptunians, we propose to use longer exposures with magnitude 
limit about $m_{V}=22$. Considering the problem with acquiring adequate data for orbit computation of these objects, follow-up astrometry, at least for some of them, will be useful.

- Cometary features

The majority of new ground-based discoveries of comets come from large NEO surveys. The first step in finding these new members of the population of cometary bodies consists in confirmatory astrometric observations along with inspection for cometary features. A timely recognition of new comets can help in planning further observation campaigns.

- Search for new asteroids

Even though our primary goal is astrometric follow-up of NEOs and other unusual objects, all of our CCD images are processed not only for target objects, but also examined for possible new object(s). This can be achieved because the effective field of view, exposure time, and the limiting magnitude of $m_{V}=22$ enable us to find many possible new object(s). Obtained images are processed with special attention to fast- and slow-moving objects.

\section{Technology}

A special software package has been developed for the KLENOT Project using a combination of programs running on Windows and Linux platforms. The system consists of observation planning tools, data-acquisition, camera control and data processing tools.

\subsection{Observations planning}

The SQL database stores information on minor planets updated on daily bases from textbased databases; the MPC Orbit Database (MPCORB), maintained by the Minor Planet Center, and from the Asteroid Orbital Elements Database (ASTORB), created and maintained by E. Bowell at the Lowell Observatory. The asteroids listed on Spaceguard system Priority List and objects listed as a Virtual Impactors by SENTRY (JPL) or by CLOMON2 (NEODyS) are flagged in the SQL database as well.

In addition the database holds orbital elements and other useful data of all solar system objects discovered at Klet and also information on comets created and updated from several sources by Klet. Positions, times, and observed objects on all of the processed plates and CCD images are also stored in the database.

For observation planning a web-based tool called ephem is used. The tool allows an observer to get ephemeris for one minor planet and/or for minor planets in a specified field at given time. The results can be sorted out to objects of given magnitude and/or type; i.e. to NEAs, PHAs, Virtual Impactors, Klet discoveries, critical list objects, unusual or distant minor planets, Trojans, Spaceguard Priority List objects and comets. The output list also includes designation, position in the sky, magnitude, and other ephemeris data, as well as information on object type, ephemeris uncertainty, date of the last observation and length of orbital arc used in orbit computation.

There is also used another tool for observation planning. Program KAC - Klet Atlas Coeli — shows stars and solar system objects together with a line showing their daily motion in a selected region on the sky. The size of the region corresponds to the FOV of the telescope and can be used to check the telescope's position during observation. The USNO-A2.0 star catalog is used as a source of positions and magnitudes of stars.

\subsection{Data processing}

Program Astrometry has been developed for astrometric measurement of CCD images. It uses from 20 to 200 reference stars from the USNO-A2.0 catalogue per measured object. 
Images are reduced and all objects with realized condition for signal to noise ratio are detected on the image; objects are then identified with catalogue and coordinates of objects are determined.

The user then selects which object on the image the output should be measured and results are written directly in the MPC format. The time of observation and other information needed for the output are derived from the data stored in the header of the image file. Information about the processed CCD image (time, filename, frame number, equatorial coordinates of the center of the frame, telescope used, exposure time, position of objects on frame, etc.) are added into the SQL database of processed CCD images.

The residuals of the measured astrometric positions are checked before providing them to the community. The calculation of residuals is based on osculating elements of the object near the current epoch, so they are acceptable mainly for the evaluation of observations. In addition the $\Delta-\mathrm{T}$ variation of the mean anomaly is determined. Checking of both residuals and the $\Delta-\mathrm{T}$ variation in mean anomaly helps verify object identification.

Program orbit is used for preliminary orbit determination. It allows a computation of preliminary orbital elements from observations for one or two nights on the assumption that an object is in perihelion, has been also developed. From observations over several nights the orbit of a new minor planet can be determined using Lagrange-Gauss method improved by variation of geocentric distances and variation of elements. Orbital elements of new discovered objects are stored in K_KLET database. We are working now on an improvement of this software for orbit determination taking into account perturbations from a N-body system. For exact determination of planetary positions the system uses The Planetary and Lunar Ephemerides DE405 provided by JPL.

\section{Results}

We review here the KLENOT Project results obtained from March 2002 to July 2006 (between 2005 May-2005 December the KLENOT Telescope was out of operation for maintenance).

\subsection{Astrometric measurements}

We have measured and sent to the Minor Planet Center 30,900 positions of 3706 objects, including 10,139 positions of 1233 NEAs, 104 of which were Virtual Impactors (VIs), and 1,922 positions of 130 comets.

The majority of measured NEAs were confirmatory observations and early followup observations of newly discovered objects presented on the NEO Confirmation Page (NEOCP) maintained by the Minor Planet Center. These observations were included in 437 Minor Planet Electronic Circulars. Especially notable are Virtual Impactors 2002 $\mathrm{MN}, 2003 \mathrm{QQ}_{47}$, (99942) Apophis $=2004 M N_{4}, 2004 \mathrm{VD}_{17}$, and $2006 \mathrm{BQ}_{6}$, as well as close Earth approachers $2003 \mathrm{SW}_{130}, 2004 \mathrm{FH}$, and $2004 \mathrm{XP}_{14}$.

\subsection{Recoveries}

In the framework of the KLENOT Project we recovered 16 NEAs including 2 PHAs, as it is presented in Table 1.

\subsection{Discoveries}

In the framework of the KLENOT Project we discovered 2 NEAs, as it is presented in Table 2. Their closest Earth approaches in discovery apparition are given in the last column. 
Table 1. Near-Earth asteroids recovered by KLENOT

\begin{tabular}{|c|c|c|c|c|c|c|}
\hline designation & $\begin{array}{l}\text { orbit } \\
\text { type }\end{array}$ & $\begin{array}{l}\text { disc. opp. } \\
\text { arc [days] }\end{array}$ & $\begin{array}{l}\text { last obs. in } \\
\text { disc. opp. }\end{array}$ & $\begin{array}{l}\text { recovery } \\
\text { date }\end{array}$ & $\begin{array}{c}\Delta-\mathrm{T} \\
{[\text { days }]}\end{array}$ & $\begin{array}{l}\text { reference } \\
\text { MPEC }\end{array}$ \\
\hline $2001 F Z_{6}$ & Amor & 76 & 2001 June 2 & 2003 Feb. 26.10 & +0.0074 & 2003-D31 \\
\hline $2001 F T_{6}$ & Aten & 26 & 2001 Apr. 18 & 2003 Mar. 25.03 & -0.15 & 2003-F37 \\
\hline $1993 F S$ & Amor & 86 & 1993 June 13 & 2003 Mar. 25.09 & +.024 & 2003-F38 \\
\hline $1999 T F_{211}$ & Apollo [PHA] & 36 & 1999 Nov. 11 & 2003 Aug. 4.01 & +1.7 & 2003-P10 \\
\hline $2001 F B_{7}$ & Amor & 118 & 2001 July 14 & 2003 Aug. 24.04 & +0.0059 & 2003-Q24 \\
\hline $2002 S R_{41}$ & Apollo & 31 & 2002 Oct. 31 & 2003 Aug. 24.88 & -0.0019 & 2003-Q31 \\
\hline $2000 R S_{11}$ & Apollo [PHA] & 290 & 2001 June 19 & 2003 Aug. 27.10 & -0.0076 & 2003-R28 \\
\hline $2002 R R_{25}$ & Aten & 56 & 2002 Oct. 30 & 2003 Sept. 5.05 & +0.0010 & 2003-R30 \\
\hline $1999 V S_{6}$ & Apollo & 181 & 2000 May 5 & 2003 Sept. 5.12 & +0.00028 & 2003-R31 \\
\hline $2001 S G_{276}$ & Amor & 56 & 2001 Nov. 21 & 2003 Sept. 5.13 & -0.025 & 2003-R34 \\
\hline $2003 L P_{6}$ & Apollo & 30 & 2003 July 12 & 2004 Feb 17.15 & +0.005 & 2004-D02 \\
\hline $2001 Y F_{1}$ & Apollo & 250 & 2002 Aug. 23 & 2004 July 16.98 & +0.0046 & $2004-\mathrm{O} 20$ \\
\hline $2003 E N_{16}$ & Amor & 145 & 2003 Apr. 27 & 2004 July 17.01 & -0.032 & 2004-O24 \\
\hline $2002 P Q_{142}$ & Apollo & 41 & 2002 Sept. 21 & 2004 Sept. 4.91 & -0.36 & 2004-R25 \\
\hline $2003 M A$ & Amor & 83 & 2003 Sept. 7 & 2005 Jan. 18.05 & +0.00000 & 2005-B29 \\
\hline $2001 G Q_{2}$ & Apollo & 14 & 2001 Apr. 28 & 2005 Mar. 31.98 & +0.009 & 2005-G09 \\
\hline
\end{tabular}

Table 2. Near-Earth asteroids discovered by KLENOT

\begin{tabular}{lccccccc}
\hline designation & orb.type & a $[\mathrm{AU}]$ & e & i [deg.] & H & observed arc & Earth approach [AU] \\
\hline $2002 \mathrm{LK}$ & Apollo & 1.10 & 0.15 & 25 & 24.2 & 2002 June 1-8 & 0.023 \\
$2003 \mathrm{UT}_{55}$ & Aten & 0.98 & 0.15 & 16 & 26.8 & 2003 Oct. 26-27 & 0.0074 \\
\hline
\end{tabular}

\subsection{Comets}

A part of Near-Earth object population consists of comets. The first step of recognizing this comet fraction is an analysis of possible cometary features of newly discovered bodies. Since March 2002 we have confirmed 28 newly discovered comets (i.e. we found cometary features of objects with unusual motion presented on the NEO Confirmation page). A natural further step should be to pursue the behavior of such cometary bodies i.e. to obtain observation data of Near-Earth comets outbursts, fragmentation, splitting and so on. In the framework the KLENOT Project we detected nucleus duplicity of comet C/2004 S1 (Van Ness) and provided astrometric measurements of 17 fragments of comet 73P/Schwassmann-Wachmann 3 including an independent detection of its several new fragments during its 2006 close approach to the Earth.

\section{Future}

The role of the follow-up in connection to the new generation of all-sky deeper surveys should be discussed. These surveys plan to provide their own follow-up, so the role of "small" observatories providing confirmations and early follow-up of NEOs will be changed. If it will be the case the role of such observatories should be moved towards NEOs which are in urgent need of astrometric positions determination over longer arc including Virtual Impactors, radar and mission targets. These changes would depend also on possible improvements made by the Minor Planet Center (MPC), SENTRY and NEODys.

Our contribution to the changing role of follow-up observatories is included in our future plans. In order to improve our possibility to observe fainter objects we plan to prepare software for co-adding of KLENOT multi-TIFF images. Secondly, in order to improve 
precision of KLENOT astrometric measurements we plan to change the USNO A2.0 astrometric catalogue to the USNO B1.0 and UCAC3 astrometric catalogues. Finally, due to the improvements planned by the MPC we have been working on incorporating of the new MPC format for astrometric observations to the Klet Software Package. These improvements will make us able to meet the new scientific and practical challenges.

\section{Outreach Activities}

Considering recent research results there is a growing interest in minor planets and comets among general public and media. Especially NEOs and the impact hazard attract much attention.

There are many interesting and well designed websites, but naturally all of them are in English. Considering that there is still an unsatisfactory level of knowledge of English language in our society, we have decided to design a NEOs website in Czech as a clear, comprehensive and up to date information about NEOs based on our long-time observing program at the Klet Observatory and our experience in education programs.

Czech Public Service on NEOs - www.planetky.cz - was designed by the Klet Observatory on February 2001. Up to now, we have published here 173 articles spread into six themes. This number includes 58 articles about Near-Earth Objects. There are following services for visitors on www.planetky.cz pages: full-text search procedure, sorting out articles into six themes, server statistics, external links to important NEO webpages all over the world, orbit diagrams of minor planets, electronic postcards, RSS Chanel, and the latest news from an additional Klet server www.komety.cz about comets.

It is been indicated (August 1, 2006) that more then 122,000 visitors has viewed more then 730,000 pages on website www.planetky.cz so far. Our website serves to general public including journalists, students and educators at different levels. On the basis of language similarities this website has also been visited by many people from Central and East European countries like Slovakia, Poland, Ukraine, Russia and others.

The system used on server www.planetky.cz was created with PHP-j00k, a web portal system based on PHP-Nuke, Free Software released under the GNU/GPL license with changes made by the Klet Observatory. Pages are displayed using PHP and mySQL.

The server www.planetky.cz has its own International Standard Serial Number ISSN 1214-6196.

\section{Acknowledgements}

The work of the Klet Observatory and the KLENOT Project is funded by the South Bohemian Region.

\section{References}

Tichá, J., Tichý, M. \& Kočer, M. 2002, ESA SP-500: ACM 2002793

Tichý, M., Tichá, J. \& Kočer, M. 2005, International Comet Quarterly 27, 87

Sekanina, Z., Tichý, M., Tichá, J. \& Kočer, M. 2005, International Comet Quarterly 27, 135

Pascu, D., et al. 2002, ASP Conf. Series 272, 361

Chamberlin, A. B., Chesley, S. R., Chodas, P. W., Giorgini, J. D., Keesey, M. S., Wimberly, R. N., \& Yeomans, D. K. 2001, Bull. Am. Astron. Soc. 33, 1116

Chesley, S. R. \& Milani, A. 1999, Bull. Am. Astron. Soc. 31, 28 